\title{
Smart Vehicle Parking System Using Computer Vision and Internet of Things (IoT)
}

\author{
O.E. Taylor, P.S. Ezekiel, and V.T. Emmah
}

\section{ABSTRACT}

Internet of Things is the interconnectivity between things, individuals and cloud administrations by means of web, which empowers new plans of action. Because of these exchanges, immense volumes of information are smartly created and is shipped off cloud-based server through web; the information is being handled and broken down, bringing about significant and convenient activities for observing the car parking. The serious issue that is arising currently at a worldwide scale and developing dramatically is the gridlock issue brought about by vehicles. A worldwide scale and developing dramatically is the gridlock issue brought about by vehicles. Among that, finding a better parking sparking space in urban areas has become a major problem with an increase of the numbers of vehicles on a daily bases. Therefore, making it difficult in having a better and safe parking spot. The system proposes an intelligent smart parking system using computer vision and internet of things. The proposed system starts by acquiring a dataset. The dataset is made up images of various vehicles, which was collected from the faculty of science car park at the Rivers State University, Port Harcourt, Rivers State Nigeria. We proposed two methods for vehicle/parking slot detection. The first method is the use of convolution neural network algorithm which is used with a haar cascade classifier in detection of multiple vehicles in a single picture and video, and put rectangular boxes on identified vehicles. This first method obtained an accuracy of $99.80 \%$. In the second method, we made use of a Mask R-CNN, here we download a pre-trained model weights which was trained on a coco dataset to identify various objects in videos and pictures. The Mask R-CNN model was used to identify various vehicles by putting a bounding box on each of the vehicle detected, but one of the problem of the Mask R-CNN is that it quite slow in training, and it could not really detect all vehicles tested on a high quality high definition video. In summary our, trained model was able to detect vehicles and parking slot on high quality video and it consumes lesser graphic card.

Keywords: computer vision, internet of things, mask R-CNN, convolutional neural network, smart parking
Published Online: April 30, 2021

ISSN: $2736-5492$

DOI : 10.24018/ejcompute.2021.1.2.12

\section{O.E. Taylor *}

Department of Computer Science, Rivers State University, Port Harcourt, Nigeria (e-mail: tayonate@yahoo.com)

P.S. Ezekiel

Department of Computer Science, Rivers State University, Port Harcourt, Nigeria (e-mail: ezekielpromise27@gmail.com) V.T. Emmah

Department of Computer Science, Rivers State University, Port Harcourt, Nigeria (e-mail: vikae2solo@yahoo.com)

\section{INTRODUCTION}

Internet of Things is the interconnectivity between things, individuals and cloud administrations by means of web, which empowers new plans of action. Because of these interchanges, immense volumes of information are smartly created, and the created information is shipped off cloudbased server through web and the information is being handled and broke down, bringing about significant and convenient activities for observing the car parking. The serious issue that is arising currently in a worldwide scale and developing dramatically is the gridlock issue brought about by vehicles. Among that, finding a better parking space in urban areas has become a major problem with an increase of the numbers of vehicles on a daily bases. Therefore, making it difficult in having a better and safe parking spot. Intelligent Parking framework acquires the data, for example, accessibility of the parking spot, when the car parked in, when the parked car drove out. It includes the keen sensors that could be fixed in streetlamps for each parking spot, assortment of ongoing information, an electronic entrance to screen the parking spots and a versatile application which will empower client to hold a parking spot and make online installment from their portable application for their stopping [1]. .

With rapid increasing number of vehicles in the course of recent years, tracking down an empty parking spot has become a significant issue for drivers in jam-packed urban areas. More than 1.3 million drivers battle each day to discover accessible parking spots in Shanghai. Additionally, looking for an empty parking spot prompts a normal of $30 \%$ of gridlock [2]. Furthermore, 945,000 additional miles are voyaged and 47,000 gallons of fuel are burned-through which produces 728 tons of carbon dioxide on normal each year in Los Angeles territory alone [3]. Thus, the thorough quest for accessible parking spots ascends to major issues, for example, gridlock, air contamination, and squandering drivers' occasions [4]. The journal reserves the right to do the final 
formatting of your paper.

Because of the headway in remote correspondences and Internet of Things (IoT) gadgets, Intelligent parking framework has been arising as a productive answer for the swiftly developing issue of finding empty parking spots. Ordinarily; in Intelligent parking framework, an IoT gadget is introduced in each parking space and uses an ultrasonic sensor to identify if a specific parking space is accessible. Thus, it gives inhabitance status of parking spots to a specialist organization. The specialist organization empowers drivers to check the accessible parking spots and reserve online spot, which encourages finding empty parking spots. Accordingly, the Intelligent parking has been conveyed in various urban communities [5]. Precise localization of vehicles in streaming videos or pictures is a critical errand for a few applications, for example, traffic controlling, parking area occupation, or urban planning. Assembling speedy vehicles data in the information pictures gives quick consideration in a clogged populace bigger zone. Without the robotized object location, the framework would require the sending of force sensors or manual work at greater expenses. Because of the fast development of vehicle proprietorship worldwide lately, vehicle security has become a worry and basic issue. The diminished expense optical gadgets [6].

This paper presents a convolutional neural network algorithm for smart vehicle parking system computer vision and internet of things. With the help of computer vision, the system will be able to recognize and detect both allocated and empty parking space.

\section{RELATED WORKS}

A Genetic Algorithm Approach to Autonomous Smart Vehicle Parking System [7] Presented a self-determining streetcar framework and a genetic methodology that will robotize vehicles at shopping center .The self-governing streetcar framework and the genetic algorithm is being actualized to track down the best parking opening, which makes the proposed framework proficient as far as space usage and streetcar productivity. The genetic methodology assisted their proposed framework with enhancing the scanning interaction for the best area absent a lot of time wastage. The Autonomous streetcar framework diminishes the holding up season of the customers in line. The client can leave their vehicle at the passage and the parking will be taken consideration by the intelligent streetcar. The lining hypothesis is applied to infer the usage, productivity and holding up time factor of the framework. The exhibition investigation chart shows that the use and proficiency factor is expanded with G.A without bargaining the decreased holding up time when contrasted with a framework without G.A. The genetic algorithm method and utilization of lining hypothesis model, helped in lightening the parking spot shortage and vehicle-booking issue.

Smart City: IOT Based Prototype for Parking Monitoring and Management System Commanded by Mobile App [1] proposed a model for giving a superior answer for intelligent city mainly concentrating on helping the general public for problem free stopping and which helps the nearby government in generating income. This model would screen the parking spots accessibility through the rising of Internet of Things (IoT) innovation and mechanize the tagging framework from advanced cells by $\mathrm{m}$ Ticketing framework.
The framework screens all the differentiated on-road parking spot states by methods for information it has from the information worker. The absolute number of parking spots, and the aggregate number of accessible parking spots, and the complete number of consumed parking spots can be checked by their proposed model.

Privacy-Preserving Smart Parking System Using Blockchain and Private Information Retrieval [5] proposed a security safeguarding Intelligent parking framework utilizing blockchain and private data recovery. Initial, a consortium blockchain was made by various parking area proprietors to guarantee security, straightforwardness, and accessibility of the parking agents. At that point, to safeguard the drivers' area security, they embrace private data recovery method to secretly recover parking agents from blockchain hubs. Likewise, a short randomizable mark was utilized to permit drivers to confirm for holding accessible stopping spaces from stopping proprietors secretly. Their framework assessments show that their proposed framework protects drivers' security with low correspondence and calculation overheads.

A Smart Parking System Based on NB-IoT and Third-party Payment Platform [8] proposed information sensor hub that will be communicated by Narrowband Internet of Things (NB-IoT) module, which is another cell innovation presented for Low-Power Wide-Area (LPWA) applications. Essential data the executives, charge the board, sensor hub observation, task the board and business knowledge modules are actualized on the cloud worker. With coordinated outsider installment stage and stopping guide administration, the versatile application created for drivers is simple and helpful to utilize. As of now, they sent their proposed framework in two urban areas to improve the usage of existing stopping offices successfully.

An IoT-based E-Parking System for Smart Cities [9] introduced a model for an E-parking framework that will give a novel parking supervision solving for different stopping office regions all through the city. The proposed E-parking framework to empower the drivers to acquire data on accessibility of parking spot and to hold some parking garage through an appropriate GUI that implies reservation based parking supervision office. This proposed framework can without much of a stretch distinguish vehicle's inappropriate parking inside the parking area and gauge the span of the parking area's inhabitance by some vehicle by utilizing an incorporated segment considered parking meter that is sent at each parking garage. Their proposed framework additionally empowers the programmed assortment of stopping charges by giving keen installment alternatives to the driver

Smart Car Parking System Solution for the Internet of Things in Smart Cities [10] proposes an intelligent vehicle parking framework that will help clients to tackle the issue of finding a parking spot and to limit the time spent in looking for the closest accessible vehicle parked. Likewise, it furnishes users with streets gridlock status. Also, the proposed framework gathers the crude information locally and extricates features by applying information sifting and combination strategies to lessen the communicated information over the organization. From that point onward, the changed information is shipped off the cloud for preparing and assessing by utilizing Machine learning techniques. 
Towards a completely computerized vehicle leaving framework [6] depicted a robotized vehicle parking framework. The proposed framework comprised of a camera introduced at the passageway/exit of the parking area. Casings were consistently gained by the camera. In the event that there was an identified face, it was enlisted in the data set. At the point when a driver was leaving, face picture was caught again at the leave terminal and contrasted against the database to finalize identity. They proposed reasonable calculations for every module and completed an impromptu test to check the attainability of the proposed framework. They notice a high precision of article location (vehicle and face) module. Nonetheless, face acknowledgment pool needs genuine consideration. Specifically, we see that AdaBoostLDA based face acknowledgment algorithm exactness isn't influenced by picture goal and handles face picture goal up to $15 \times 15$ pixels.

Towards a completely computerized vehicle leaving framework [6] depicted a robotized vehicle parking framework. The proposed framework comprised of a camera introduced at the passageway/exit of the parking area. Casings were consistently gained by the camera. In the event that there was an identified face, it was enlisted in the data set. At the point when a driver was leaving, face picture was caught again at the leave terminal and contrasted against the database to finalize identity. They proposed reasonable calculations for every module and completed an impromptu test to check the attainability of the proposed framework. They notice a high precision of article location (vehicle and face) module. Nonetheless, face acknowledgment pool needs genuine consideration. Specifically, we see that AdaBoostLDA based face acknowledgment algorithm exactness isn't influenced by picture goal and handles face picture goal up to $15 \times 15$ pixels.

IoT based Parking System utilizing Google [11] built up an intelligent parking android gadget that will utilize Internet of Things(Iot). The android application has an easy to understand interface that can give better outcomes for parking places albeit the Google maps are the vital component of this framework. From these guides client can find the accessible spaces as well as can discover the heading for it. Their framework showed the proficient spot for parking and it will likewise direct to reach there. This can lessen mishaps just as contamination.

Low Cost Smart Parking System for Smart Cities [12] built up an online based stopping booking and the executives framework to address the stopping issues in Hyderabad city and for organization in Smart Cities. The users can book stopping space whenever and from any area with their Mobile Phone or with a Computer. The client questions for the accessibility of empty openings and the framework checks the Database for the presence of free spaces. The framework shows the quantity of free spaces and the client is incited to choose an opening and continue for installment to book the ideal opening. After the affirmation of installment, the information base is refreshed, and a standardized identification is created for the client. Each stopping opening is fitted with a ultrasonic sensor, which checks the situation with the stopping space (Occupied or Vacant). The three ultrasonic sensors (HCSR04) are interfaced with ESP8266 based NodeMcu board. Esp8266 has 3.3V open minded pins.
Subsequently, reverberation pin of HC-SR04 is associated with Esp8266 by means of resistor network shaped utilizing $2.2 \mathrm{~K}$ and $4.7 \mathrm{~K}$. Here $2.2 \mathrm{~K}$ is associated with Echo pin, and $4.7 \mathrm{~K}$ is grounded.

\section{MethodOLOGY}

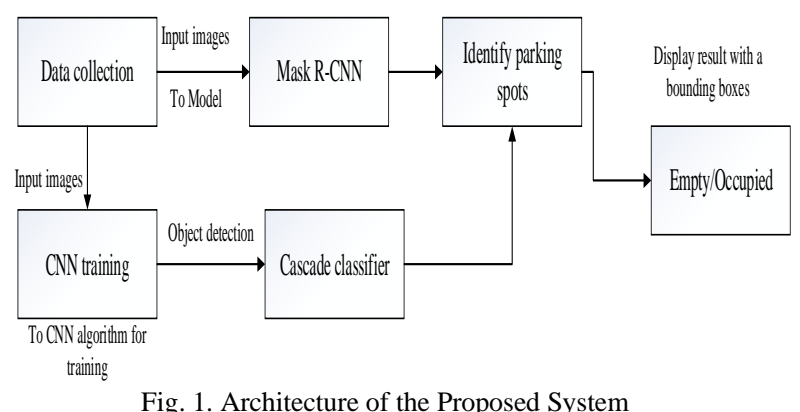

Data Collection: The proposed system uses a smart vehicle dataset, which was collected from the Rivers State University, Port-Harcourt, Nigeria. This dataset consisted of car images, which are packed at the science car park of the Rivers State University. The dataset was categorized into two. which is a training data and a testing data. The training data contains $70 \%$ of the captured images while the testing data contains $30 \%$ of the captured images. The images was inputted into The mask R-CNN model and our proposed CNN model as input data.

Convolutional Neural Network (CNN): Our proposed Convolutional Neural Network Algorithm will be build using a total layer of four with two hidden layers and one output layer. The parameters used in building the proposed model are : target_size $=(150,150)$, batch_size $=128$, activation $=$ relu, for the first layer, wile for the last layer, activation = softmax. Optimizer $=$ RMSpro, epoch $=9$.

Mask R-CNN: Mask R-CNN is a state-of-the-art model for instance segmentation, developed on top of a faster R-CNN [13]. Figure 2 shows the architecture of a Mask R-CNN with different stages. The stages can be described as follows:

1) Stage 1: The primary stage comprises of two networks, spine (ResNet, VGG, Inception, and so forth) and region proposition network. These networks run once per picture to give a bunch of region recommendations. Region proposition are areas in the component map, which contain the object.

2) Stage 2: In the subsequent stage, the organization predicts bounding boxes and object class for every one of the proposed area acquired in stage1. Each proposed region can be of various size while completely associated layers in the organizations consistently require fixed size vector to make expectations. Size of these proposed districts is fixed by utilizing either RoI pool (which is very much like MaxPooling) or RoIAlign technique. 


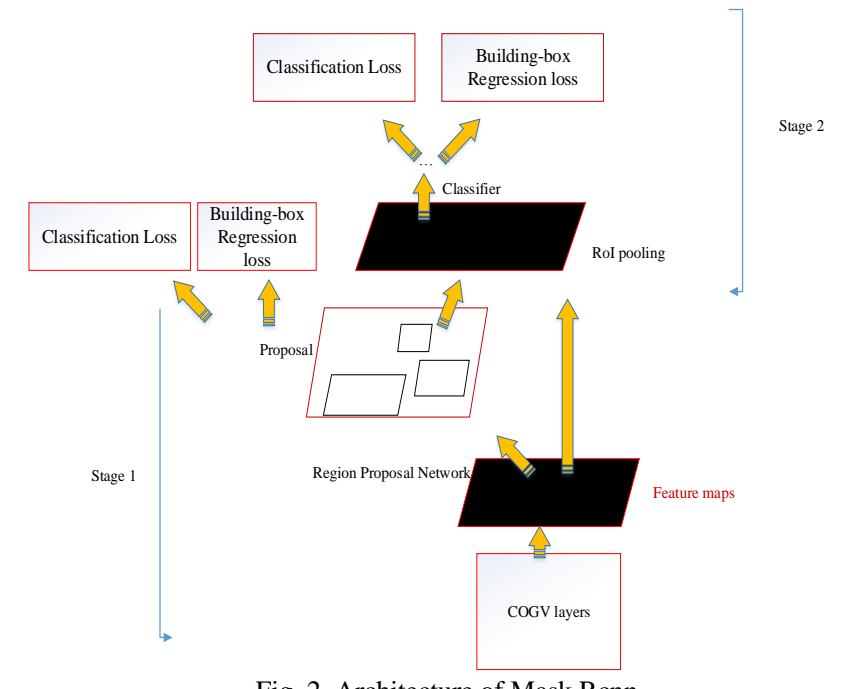

Fig. 2. Architecture of Mask Rcnn

Cascade Classifier: This is a class in computer vision (OpenCv) to detect objects in videos in video stream. Cascade classifier is a machine learning based approach where a cascade function is trained from a lot of positive and negative images. It is used to detect objects in other images. We will be using cascade classifiers to detect multiple vehicles in an image. Figure 3 shows the architecture of a cascade classifier.

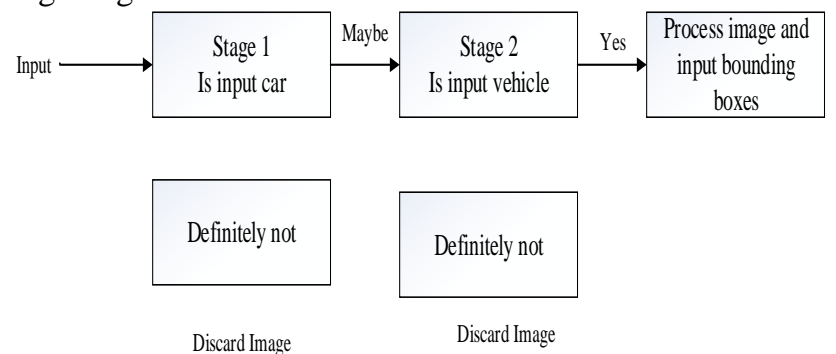

Fig. 3. Training Cascade Architecture

\section{ALGORITHM IN CASCADE CLASSIFIER TRAINING}

Step1: Users selects values for $f$, the maximum acceptable false positive rate per layer and $d$ the maximum acceptable detection rate per layer.

Step2: Use selects target overall false positive rate, Ftarget.

Step3: $\mathrm{P}=$ set of positive examples

Step4: $\mathrm{N}=$ set of negative examples

Step5: F0 = 1.0: D0 = 1.0

Step6: $i=0$

Step7: while Fi > Ftarget

$$
-\mathrm{i} \leftarrow \mathrm{i}+1
$$$$
- \text { ni }=0 ; \mathrm{Fi}>\mathrm{Fi}-1
$$$$
\text { - while } \mathrm{Fi}>\mathrm{f} \times \mathrm{Fi}-1
$$

$$
* \text { ni } \leftarrow \text { ni }+1
$$

* Use $\mathrm{P}$ and $\mathrm{N}$ to train a classifier with ni features using AdaBoost

* Evaluate current cascade classifier on validation set to determine Fi and Di .

* Decrease threshold for the ith classifier until the current cascade classifier has a $\times$ Di -1 (this also affects Fi) detection rate of at least $\mathrm{d}$

$$
-\mathrm{N} \leftarrow \varnothing
$$

- If Fi > Ftarget then evaluate the current cascade detector o the set of non-vehicle images and put any false detection into the set $\mathrm{N}$
Identify parking space: Our proposed model will be able to identify various vehicles from the science car park from rivers state university, and it will also be able to identify some empty parking space at the faculty of science car park.

\section{RESULT AND DISCUSSION}

Our system proposes an Intelligent system for smart parking system using computer vision and internet of things. The proposed system starts by acquiring a dataset. The dataset is made up images of various vehicles, which was collected from the faculty of science car park at the Rivers State University, Port Harcourt, Nigeria. We divided the images into a training folder and a testing folder. These images were labelled using VGG image annotation tool, which we used in defining the region of our collected images for both training images and testing images. We labelled each of the images individually in other to have a better description of this images. We labelled multiple objects in each of the images by drawing a rectangle and writing down a description of the objects in the image. After successfully labelling of this images, we imported some python libraries (numpy, pandas, tensorflow, cv2, matplotlib.pyplot, and os). We created a directory for these images and we used cv2.imread in reading the images from their directory path. After set directory path and reading the images from directory, we then passed the mentioned images to our proposed model. We proposed two algorithms for the identification of vehicles and empty parking space. The first model was built using convolutional neural network with a total of four layers, two hidden layers and one output layer. Our proposed model uses the following parameters: target_size $=(150,150)$, batch_size $=128$, activation $=$ relu, for the first layer, wile for the last layer, activation $=$ softmax. Optimizer $=$ RMSpro, epoch $=9$. After successful compiling of the convolutional neural network algorithm, we had an accuracy of about $99.80 \%$. This can be seen in figure 7 . For testing of our proposed model to detect multiple vehicles and multiple parking space on a single image or on a live streaming video, we used Haar cascade classifier. With haar cascade classifier, we were able to draw multiple rectangular boxes on live streaming videos and also on multiple objects in an image. The second algorithm has to do with a pre-trained model weight of a Mask R-CNN algorithm, which will, downloaded. This pre-trained model has already been trained on a coco dataset for object detection. For successful implementation of the pre-trained Mask R-CNN model, we installed some dependences and we cloned an environment and configured all paths and directories. After successful configurations and setting of paths, we implmeneted the pre-trained Mask R-CNN model and used it in identifying vehicles and empty parking space. Figure 5 shows the model compilation which has to do with the total trainable parameters used in out proposed convolutional neural network model. Figure 6 shows the number of training steps, the time taken to complete one training steps and the loss and accuracy values at each training steps. Figure 7 and 8 shows a graphical representation of the accuracy and loos values at each training epoch. Figure 9 shows the result of our proposed convolutional neural network algorithm when we tasted it on picture taken on the faculty of science car park at the River State university, Port Harcourt Nigeria, where the model 
detected various vehicles which it drew with a rectangular box of colour red. Figure 10 shows the result of our proposed convolutional neural network algorithm on a custom image downloaded from google.com, where it detected both vehicles and empty vehicle space. Figure 11 shows the result of the Mask R-CNN on a custom image downloaded from google.com

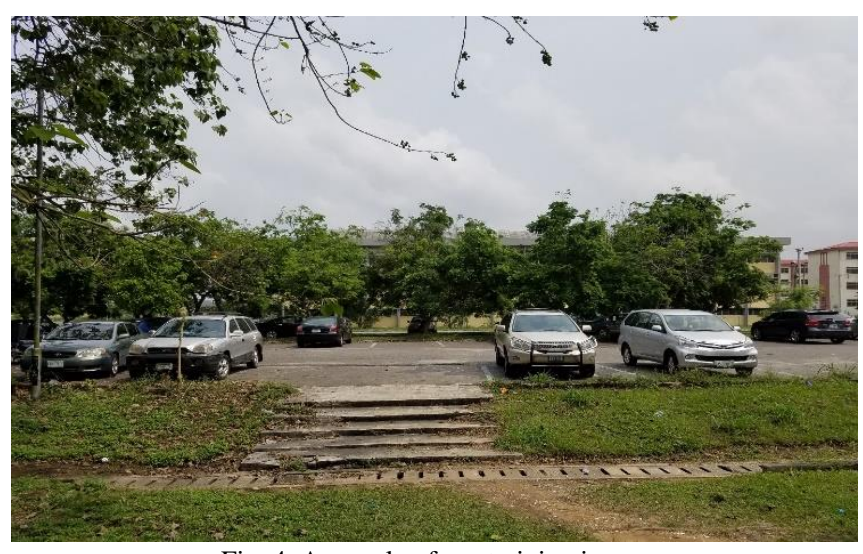

Fig. 4. A sample of our training image

\begin{tabular}{|c|c|c|}
\hline \multicolumn{3}{|l|}{ mode1. summary () } \\
\hline Model: "sequential" & & \\
\hline Layer (type) & Output Shape & Param \# \\
\hline conv2d (Conv2D) & $\begin{array}{l}=================== \\
\text { (None, } 148,148,32)\end{array}$ & 896 \\
\hline max_pooling2d (MaxPooling2D) & (None, 74, 74, 32) & 0 \\
\hline Conv2d_1 (Conv2D) & (None, 72, 72, 32) & 9248 \\
\hline max_pooling2d_1 (MaxPooling2 & (None, 36, 36, 32) & 0 \\
\hline flatten (Flatten) & (None, 41472) & 0 \\
\hline dense (Dense) & (None, 64) & 2654272 \\
\hline dense_1 (Dense) & (None, 2) & 130 \\
\hline
\end{tabular}

Fig. 5. Our proposed convolutional neural network model summary.

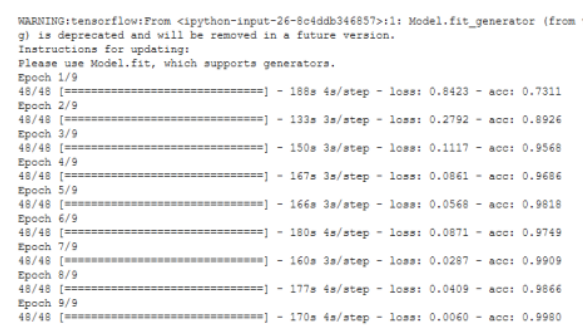

Fig. 6. This shows the number steps per training epoch with the losses and accuracies at each epoch during training.

Training accuracy with epochs

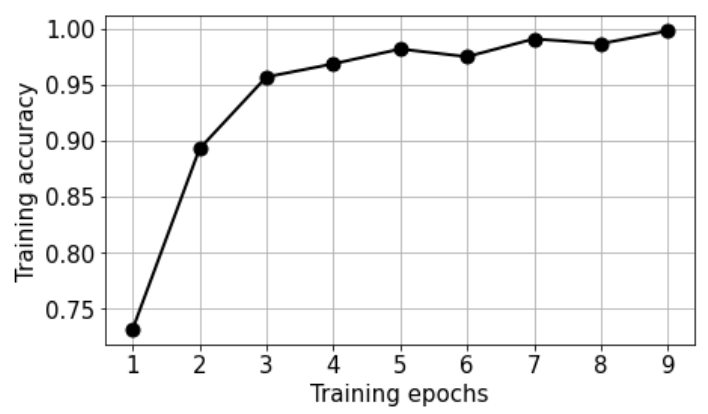

Fig. 7. Showing accuracy vs training epoch
Training loss with epochs

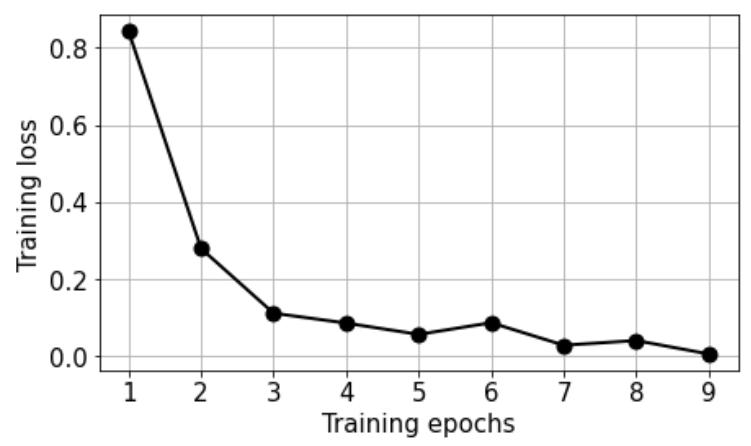

Fig. 8. Showing the loss values during the training process

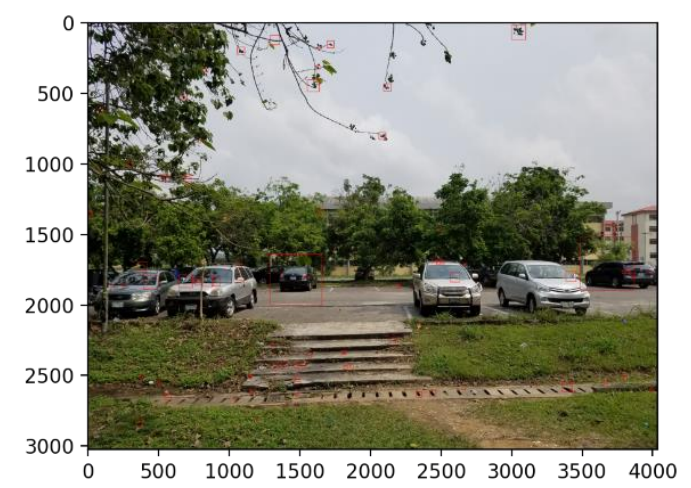

Fig. 9. The system detected various vehicles by drawing a rectangular box on them.

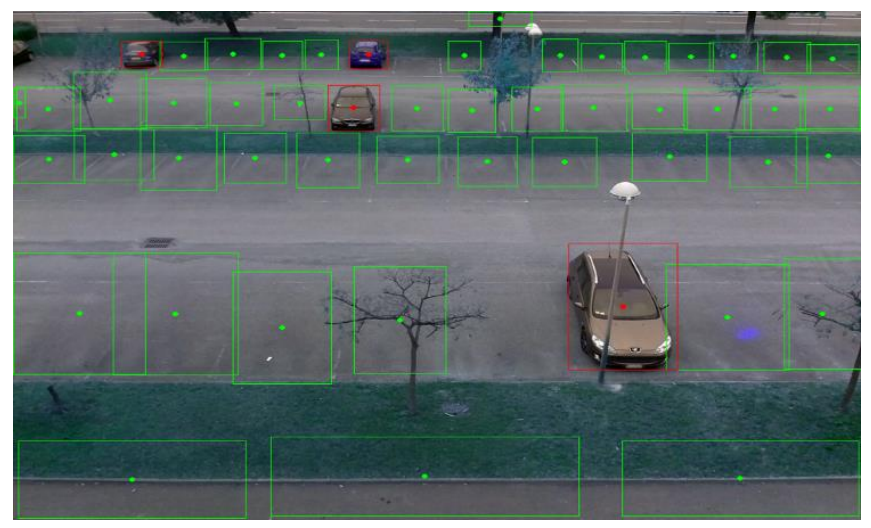

Fig. 10. Here our proposed model detected an empty space with a green rectangular box and a red rectangular box for a vehicle

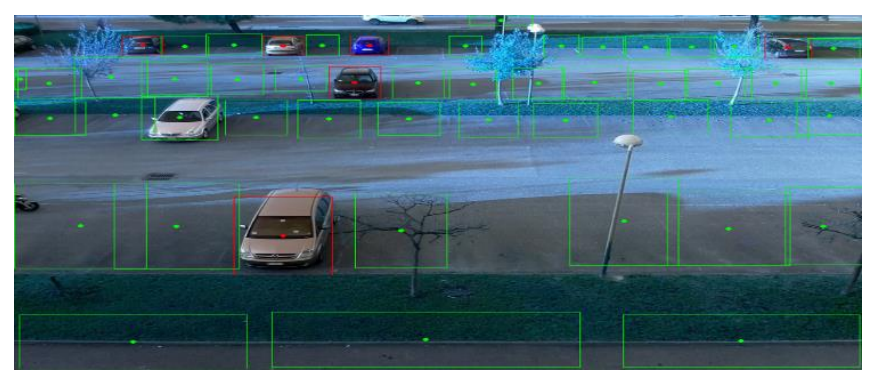

Fig. 11. Result of the pre-trained Mask R- CNN model detected an empty space with a green rectangular box and a red rectangular box for a vehicle

\section{CONCLUSION AND FUTURE WORK}

Internet of Things is the interconnectivity between things, individuals and cloud administrations by means of web, which empowers new plans of action. Because of these interchanges, immense volumes of information are smartly created, and the created information is shipped off cloud- 
based server through web and that information is being handled and broke down, bringing about significant and convenient activities for observing the car parking. Finding the best parking space in Port Harcourt, Rivers State Nigeria have resulted to many critical issues, some end up parking wrongly which in turn lead to vehicle congestion, therefore causing little traffic jam. The serious issue that is arising currently at a worldwide scale and developing dramatically is the gridlock issue brought about by vehicles. Finding a better parking space will be very important for most Nigerians, therefore developing a smart parking system that will notify users of available space will be very useful. In this paper, we proposed an intelligent system for smart car parking using Internet of this and computer vision. We proposed two methods for vehicle/parking slot detection. This first method was the use of convolution neural network algorithm which we used with a haar cascade classifier in detection multiple vehicles in a single picture and videos. We achieve an accuracy of $99.80 \%$. The second method was making use of a Mask R-CNN, here we download a pre-trained model which was trained on a coco dataset to identify various objects in videos and images. We identify various vehicles and parking slot on a single image using this model. This work can further be extended by using a live cctv camera that will be mounted on a car parking garage, and notify users who parked in correctly when there is available parking slot.

\section{APPENDIX}

Appendixes, if needed, appear before the acknowledgment.

\section{ACKNOWLEDGMENT}

The preferred spelling of the word "acknowledgment" in American English is without an "e" after the "g." Use the singular heading even if you have many acknowledgments. Avoid expressions such as "One of us (S.B.A.) would like to thank ...." Instead, write "F. A. Author thanks ...." Sponsor and financial support acknowledgments are placed in the unnumbered footnote on the first page.

\section{REFERENCES}

[1] D. D. TJB, A. Subramani, V. K. Solanki "Smart City: IOT Basel Prototype for Parking Monitoring and Management System Commanded by Mobile App", Proceedings of the Second International Conference on Research in Intelligent and Computing in Engineering (2017); pp. 341-343.

[2] T. Giuffrè, S. M. Siniscalchi, and G. Tesoriere, "A novel architecture of parking management for smart cities," Procedia-Social and Behavioral Sciences, vol. 53, pp. 16-28, 2012.

[3] D. C. Shoup, "Cruising for parking," Transport Policy, vol. 13, no. 6, pp. 479-486, 2006.

[4] H. Li, K. Ota, and M. Dong, "Network virtualization optimization in software defined vehicular ad-hoc networks," in proceeding of IEEE 84th Vehicular Technology Conference (VTC-Fall), 2016.

[5] W. A. Amiri, M. Baza, K. Banawan, M. Mahmoud, W. Alasmary, K. Akkaya "Privacy-Preserving Smart Parking System Using Blockchain and Private Information Retrieval" 2019 International Conference on Smart Applications, Communications and Networking (SmartNets), pp.1-6, 2019.

[6] Z. Mahmood, O. Haneef, N. Muhammad, S. Khattak "Towards a fully automated car parking system", IET Intelligent Transport Systems, vol.13, issue.2, pp.293-302 2018.

[7] D. Thomas, B. C. Kovoor "A Genetic Algorithm Approach to Autonomous Smart Vehicle Parking System" 6th International Conferences on Smart Computing and Communications, pp.68-76 2017.
[8] J. Shi, L. Jin, J. Li, Z. Fang "A Smart Parking System Based on NBIoT and Third-party Payment Platform" 17th International Symposium on Communications and Information Technologies (ISCIT), 2017.

[9] P. Sadhukhan "An IoT-based E-Parking System for Smart Cities" 2017 International Conference of Advances in Computing, Communications and Informatics, pp.1062-1066 2017

[10] W. Alsafery, B. Alturki, S. Reiff-Marganiec, K. Jambi "Smart Car Parking System Solution for the Internet of Things in Smart Cities", 2018 Ist International Conference on Computer Applications \& Information Security, pp.1-5 2018.

[11] S. Shinde, A. Patil, S. Chavan "IoT based Parking System using Google", International conference on I-SMAC (IoT in Social, Mobile, Analytics and Cloud), pp.634-636 2017.

[12] D.Vakula, Y. K. Kolli "Low Cost Smart Parking System for Smart Cities", Proceedings of the International Conference on Intelligent Sustainable Systems (ICISS 2017) IEEE Xplore Compliant - Part Number:CFP17M19-ART, ISBN:978-1-5386-1959-9

[13] H. Kaiming, G. Gkioxari, P. Dollár, R. Girshick: "Mask R-CNN", 2017; http://arxiv.org/abs/1703.06870 arXiv:1703.06870.

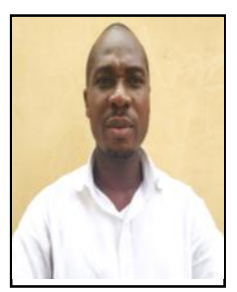

Dr. O. E. Taylor, obtained his B.Sc, M.Sc and Ph.D degrees all in Computer Science from the Rivers State University of Science and Technology, University of Ibadan and University of Port Harcourt, Nigeria respectively. He is currently Senior Lecturer in the Department of Computer science, Rivers State University, Port Harcourt, Nigeria. He is a chartered member of the Computer Professionals (Registration Council) of Nigeria and Nigeria Computer Society. His research focuses on intelligent systems, smart space, context-aware systems, machines learning algorithms and artificial intelligence. He has over ten years of teaching and research experience.

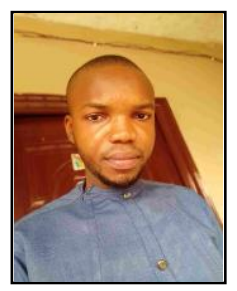

Mr. P E Ezekiel, obtained his Bachelor of Science degree from Department of Computer Science, Rivers State University. He has published four research papers in international journals which are available online. His main research work focuses on Machine Learning, Data Science, Deep Learning and Artificial Intelligence.

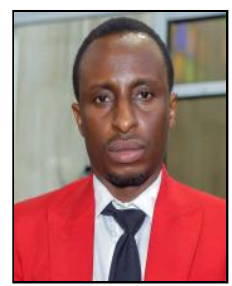

Mr. V. T Emmah, obtained his Bachelor of Science degree and Master of Science degree from Department of Computer Science, Rivers State University and University of Port Harcourt respectively. He is currently pursuing a $\mathrm{Ph} . \mathrm{D}$ in Computer Science at the University of Port Harcourt. He is a member of the Computer Professional (Registration Council) of Nigeria. His main research work focuses on Machine Learning, Data Science, Deep Learning and Artificial Intelligence. 